Copyright by the Acoustical Society of America. Moawad, M. F., Shaarawi, A. M. \& Besieris, I. M. (2004). Characterization of a nonrigid sphere using the backscattered fields of acoustic X waves. Journal of the Acoustical Society of America, 115(6), 2937-2946. doi: 10.1121/1.1715111

\title{
Characterization of a nonrigid sphere using the backscattered fields of acoustic $\mathbf{X}$ waves
}

\author{
Maged F. Moawad and Amr M. Shaarawia) \\ The Physics Department, The American University in Cairo, P.O. Box 2511, Cairo 11511, Egypt \\ loannis M. Besieris \\ The Bradley Department of Electrical and Computer Engineering, Virginia Polytechnic Institute \\ and State University, Blacksburg, Virginia 24061
}

(Received 24 September 2003; revised 26 February 2004; accepted 2 March 2004)

\begin{abstract}
The scattering of acoustic ultra-wideband X-wave pulses by a nonrigid sphere is simulated for purposes of material identification and characterization. Using the backscattered spectrum of the $\mathrm{X}$-wave pulses, a procedure is described for estimating the radius, speed of sound, and density of the sphere. The effectiveness of the suggested technique is verified in the case that the peak of the $\mathrm{X}$ wave is incident on the centers of the sphere, as well as for the off-center incidence case. (C) 2004 Acoustical Society of America. [DOI: 10.1121/1.1715111]
\end{abstract}

PACS numbers: 43.40.Fz, 43.20.Fn. [ANN]

Pages: 2937-2946

\section{INTRODUCTION}

The scattering of acoustic ultra-wideband pulses from spheres has important applications in fields such as highresolution imaging, remote sensing, material characterization, and detection of buried objects. Localized waves, one class of ultra-wideband pulses, have distinct advantages in such applications. A study along these lines has dealt with the scattering of an $\mathrm{X}$ wave, a specific class of localized waves, from a circular disk in free space or buried in the ground. ${ }^{1}$ The analysis used in that investigation was based on high-frequency techniques in combination with a pulsed plane wave representation of the X-wave solution. ${ }^{2}$ In spite of the effectiveness of the methods used in the aforementioned study, the work did not address the possibility of using the scattered signal in order to identify or characterize the scattering object. An important theoretical and experimental study of the applicability of localized waves for identification purposes was undertaken by Power, Donnelly, and MacIsaac, ${ }^{3}$ who demonstrated the possibility of identifying the radii of various types of spheres from the spectrum of the backscattered signal; the latter arose from an incident modified power spectrum (MPS) pulse. ${ }^{4}$ Their analysis considered acoustical scattering from nonrigid spheres. However, the underlying method was applicable only to the identification of the radii of the spheres, and could not be used to find the speed of sound or the density of the scattering material.

Our aim in this work is to investigate the possibility of using the spectrum of the backscattered acoustical $X$ waves in order to determine the characteristic properties of the materials of the spheres. By simulating the scattered signals from different spheres, it is demonstrated that an accurate estimate of the radii of the spheres, as well as the densities and the sound speeds of their materials, can be obtained. It is important to emphasize that the different characteristic properties of the spheres are identified using different portions of

\footnotetext{
a) On leave from the Department of Engineering Physics and Mathematics, Faculty of Engineering, Cairo University, Giza 12211, Egypt.
}

the ultra-wideband spectrum of the backscattered acoustic X wave. Consequently, the ideas considered in this paper are relevant for other ultra-wideband pulses. Nevertheless, $\mathrm{X}$ waves have the additional advantage that their extended localization range ensures that a relatively large amount of energy reaches the scattering spheres. The plan of this work is as follows. A spectral representation of the incident $\mathrm{X}$-wave is provided in Sec. II. The scattered field is deduced in Sec. III and several features of the backscattered spectrum are considered in Sec. IV. A detailed portrayal of the proposed identification scheme based on a simulation of backscattered spectra from six different materials is carried out in Sec. V. Concluding remarks are made in Sec. VI.

\section{THE INCIDENT ACOUSTICAL X-WAVE PULSE}

A spectral approach will be used to obtain the scattered field due to an incident acoustical X wave (AXW). Thus, it is important to be able to choose the spectral representation that is best suited to the adopted analysis. The AXW can be represented as a Fourier superposition over plane waves whose wave vectors form a conic surface, ${ }^{5}$ as shown in Fig. 1. The series solution of a plane wave scattered from a sphere ${ }^{6}$ can then be integrated over the Fourier spectrum of the X-wave solution in order to obtain the AXW scattered field.

To determine the AXW Fourier representation, consider the three-dimensional scalar wave equation

$$
\left(\vec{\nabla}^{2}-\frac{1}{c^{2}} \frac{\partial^{2}}{\partial t^{2}}\right) p(\vec{r}, t)=0
$$

for the pressure field; the latter can be related to a potential function $\Psi(\vec{r}, t)$ as follows:

$$
p(\vec{r}, t)=-\rho_{0} \frac{\partial}{\partial t} \Psi(\vec{r}, t) .
$$

The density of the surrounding medium is denoted by $\rho_{0}$. The AXW potential of the incident pulse, which is also a solution to the 3D scalar wave equation, can be represented in terms of the Fourier superposition 


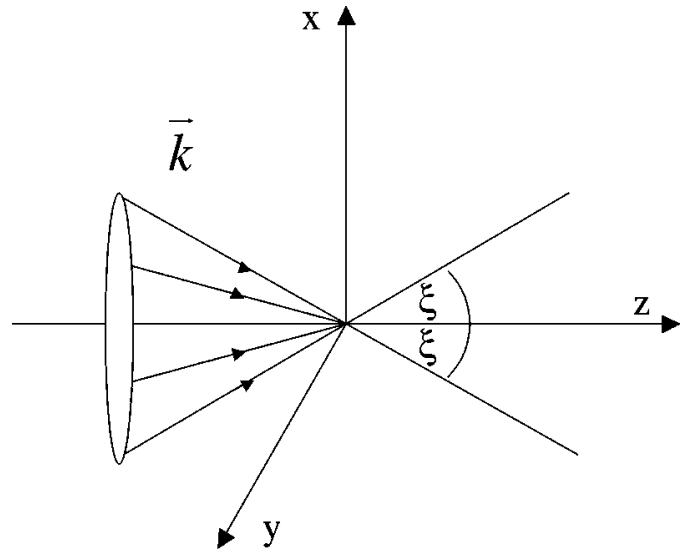

FIG. 1. Wave vectors of the normally incident $\mathrm{X}$-wave lying on a conic surface having an apex angle $\xi$.

$$
\begin{aligned}
\Psi_{\mathrm{AXW}}^{i}(\vec{r}, t)= & \int_{R^{3}} d^{3} \vec{k} \int_{0}^{\infty} d(\omega / c) e^{-i \vec{k} \cdot \vec{r}} \\
& \times e^{i \omega t} \hat{\psi}(\vec{k}, \omega) \delta(\omega-|\vec{k}| c),
\end{aligned}
$$

in which the AXW spectrum has the following specific form: ${ }^{7}$

$$
\hat{\psi}(\vec{k}, \omega)=i e^{-(\omega / c) a} \frac{2 \pi}{c \rho_{0} \sin \xi} k^{q-2} \delta\left(\theta_{k}-\xi\right)
$$

in a spherical coordinate system $\vec{k}=\left(k, \theta_{k}, \phi_{k}\right)$. The fixed angle $\xi$ is referred to as the axicon angle. One should note that the spectrum given in Eq. (3b) restricts the Fourier spectral components to plane waves propagating along directions forming a conical surface defined by the conical angle $\xi$. The substitution of the spectrum $\hat{\psi}(\vec{k}, \omega)$ in Eq. (3a) yields

$$
\begin{aligned}
\Psi_{\mathrm{AXW}}^{i}(\vec{r}, t)= & \int_{0}^{\infty} d k \int_{0}^{\pi} d \theta_{k} \int_{0}^{2 \pi} d \phi_{k} k^{2} \sin \theta_{k} \frac{i 2 \pi}{c \rho_{0} \sin \xi} \\
& \times k^{q-2} \delta\left(\theta_{k}-\xi\right) e^{-k a} e^{-i \vec{k} \cdot \vec{r}} e^{i k c t}
\end{aligned}
$$

Integrating over $\theta_{k}$ and $\phi_{k}$, we obtain

$$
\Psi_{\mathrm{AXW}}^{i}(\vec{r}, t)=\frac{i 4 \pi^{2}}{c \rho_{0}} \int_{0}^{\infty} d k k^{q} e^{-k a} e^{-i \vec{k} \cdot \vec{r}} e^{i k c t} .
$$

For integer values of $q$, the integration over $k$ in the axisymmetric (with respect to the $z$ axis) incident $\mathrm{X}$-wave pulse, viz.,

$$
\begin{aligned}
\Psi_{\mathrm{AXW}}^{i}(\rho, z, t)= & \frac{i 2 \pi}{c \rho_{0}} \frac{\partial^{q}}{\partial a^{q}} \\
& \times\left\{\rho^{2} \sin ^{2} \xi+[a+i(z \cos \xi-c t)]^{2}\right\}^{-1 / 2}
\end{aligned}
$$

Here, $\rho$ denotes the radial variable in cylindrical coordinates, the positive parameter $a$ determines the width of the pulse, and $q$ characterizes the order of the $\mathrm{X}$ wave. The axial and lateral widths of the AXW pulse are equal to $a / \cos \xi$ and $a / \sin \xi$, respectively. ${ }^{1}$ The spectral bandwidth is determined by the two parameters $a$ and $q$. The peak of the spectrum occurs at $\omega_{\text {peak }}=q c / a$. The maximum frequency correspond- ing to the $1 / e^{4}$ point is given by the expression $\omega_{\max }=(q$ $+4) c / a$. The AXW pulse, given in Eq. (5), has a highintensity central portion buried in an extended sparse background field. The localized central portion of the $\mathrm{X}$ wave has lateral and axial waists equal to $a / \sin \xi$ and $a / \cos \xi$, respectively. For an AXW pulse generated from a source having a diameter equal to $D$, the peak of the pulse travels without any dispersion to a distance $D / 2 \tan \xi$ from the source.

\section{THE SCATTERED X-WAVE PULSE}

Consider an AXW pulse incident on a sphere of an unknown material immersed in a fluid (e.g., water), where the center of the sphere is situated at the origin. In order to calculate the scattered AXW pulse, we shall consider the general expression for a plane wave incident in a direction specified by a propagation vector $\vec{k}=\left(k, \theta_{k}, \phi_{k}\right)$. Assuming a harmonic time dependence of the form $\exp (i \omega t)$, the spatial part of the plane-wave solution appearing in Eq. (4) is given by

$$
\Phi^{i}(r, \phi, \theta)=e^{-i \vec{k} \cdot \vec{r}}=e^{-i k r \cos \gamma},
$$

where $\gamma$ denotes the angle between the position vector $\vec{r}$ $=(r, \theta, \phi)$ and the wave vector $\vec{k}=\left(k, \theta_{k}, \phi_{k}\right)$. The specific choice $\theta_{k}=\xi$, required in Eq. (4), results in the angle $\gamma_{k}$ defined by the relationship $\cos \gamma_{k}=\cos \xi \cos \theta$ $+\sin \xi \sin \theta \cos \left(\phi_{k}-\phi\right)$.

Following the standard technique for calculating the scattering of plane waves from spheres, the incident plane wave is expanded in terms of Legendre polynomials and spherical Bessel functions, viz., ${ }^{8}$

$$
\begin{aligned}
\Phi^{i}(r, \theta, \phi, t)= & e^{-i k r \cos \gamma_{k}} e^{i k c t} \\
= & \sum_{n=0}^{\infty}(-i)^{n}(2 n+1) \\
& \times P_{n}\left(\cos \gamma_{k}\right) j_{n}(k r) e^{i k c t} .
\end{aligned}
$$

The function $P_{n}\left(\cos \gamma_{k}\right)$ can be separated according to the expression

$$
\begin{aligned}
P_{n}\left(\cos \gamma_{k}\right)= & \sum_{m=0}^{n} \epsilon_{m} \frac{(n-m) !}{(n+m) !} P_{n}^{m}(\cos \theta) P_{n}^{m}(\cos \xi) \\
& \times \cos \left[m\left(\phi_{k}-\phi\right)\right],
\end{aligned}
$$

where $\epsilon_{m}=1$ for $m=0$ and $\epsilon_{m}=2$ for $m \neq 0$.

The scattered field is usually represented as a series of concentric spherical waves diverging from the scatterer by means of the mathematical expression

$$
\Phi^{s}(r, \theta, \phi, t)=\sum_{n=0}^{\infty} A_{n} P_{n}\left(\cos \gamma_{k}\right) h_{n}^{(2)}(k r) e^{i k c t}
$$

where $h_{n}^{(2)}$ denotes a spherical Hankel function. The field inside a nonrigid sphere can be represented as follows:

$$
\Phi^{i n}(r, \theta, \phi, t)=\sum_{n=0}^{\infty} C_{n} P_{n}\left(\cos \gamma_{k}\right) j_{n}\left(k_{e} r\right) e^{i k_{e} c t} .
$$

The coefficients $A_{n}$ and $C_{n}$ in Eqs. (9) and (10) are determined from the appropriate boundary conditions. How- 
ever, we shall only be interested in the former for evaluating the backscattered field. At the surface of a sphere of radius $R$, the boundary conditions are chosen such that the normal component of the fluid velocity at the surface of the sphere is the same as that of the surface proper and that the pressure on the two sides of the interface between the fluid and the sphere is a continuous function. These two conditions can be written explicitly as

$$
\begin{aligned}
& \nu_{n 1}(r=R, \theta, \phi, t)=\nu_{n 2}(r=R, \theta, \phi, t), \\
& \vec{\nu}(\vec{r}, t) \equiv \vec{\nabla} \Phi(\vec{r}, t),
\end{aligned}
$$

and

$$
\begin{aligned}
& p_{1}(r=R, \theta, \phi, t)=p_{2}(r=R, \theta, \phi, t), \\
& p(\vec{r}, t) \equiv-\rho_{0} \frac{\partial}{\partial t} \Phi(\vec{r}, t) .
\end{aligned}
$$

Utilizing these boundary conditions, one obtains

$$
A_{n}=-(-i)^{n}(2 n+1) \frac{j_{n}^{\prime}(k R)+i \alpha_{n} j_{n}(k R)}{h_{n}^{(2) \prime}(k R)+i \alpha_{n} h_{n}^{(2)}(k R)},
$$

where $\alpha_{n}=i\left(\rho_{0} c / \rho_{e} c_{e}\right)\left[j_{n}^{\prime}\left(k_{e} R\right) / j_{n}\left(k_{e} R\right)\right]$. Here, $\rho_{0}$ is the density of the medium surrounding the sphere, while $\rho_{e}$ is the density of the sphere. Similarly, $c$ and $c_{e}=\sqrt{B \rho_{e}}$ $=\sqrt{1 /\left(\rho_{e} \kappa_{e}\right)}$ are the speeds of wave propagation outside and inside the sphere, respectively. The latter is expressed in terms of the density of the sphere and the adiabatic compressibility $\kappa_{e}$, which, in turn, is the reciprocal of the bulk modulus $B$. Finally, $k=\omega / c$ and $k_{e}=\omega / c_{e}$ are the wave numbers outside and inside the sphere, respectively.

If the center of the scattering sphere lies on the axis of propagation of the AXW pulse, the problem is azimuthally symmetric; thus $m=0$ is the only surviving term and the scattered potential becomes

$$
\begin{aligned}
\Psi_{\mathrm{AXW}}^{s}( & r, \theta, \phi, t) \\
= & \frac{i 4 \pi^{2}}{c \rho_{0}} \int_{0}^{\infty} d k k^{q} e^{-k a} e^{i k c t} \sum_{n=0}^{\infty}-(-i)^{n}(2 n+1) \\
& \times \frac{j_{n}^{\prime}(k R)+i \alpha_{n} j_{n}(k R)}{h_{n}^{(2) \prime}(k R)+i \alpha_{n} h_{n}^{(2)}(k R)} h_{n}^{(2)}(k r) \\
& \times P_{n}(\cos \theta) P_{n}(\cos \xi) .
\end{aligned}
$$

Consider, on the other hand, an AXW incident off-center on the sphere. Specifically, an AXW moving along the $z$ direction with its axis of propagation shifted to a line parallel to the $z$ axis and passing through the point $x=x_{0}$ and $y=0$. The incident AXW will be the same as given in Eq. (4a), but with $x$ replaced by $x-x_{0}$. In this case, the scattered potential assumes the form

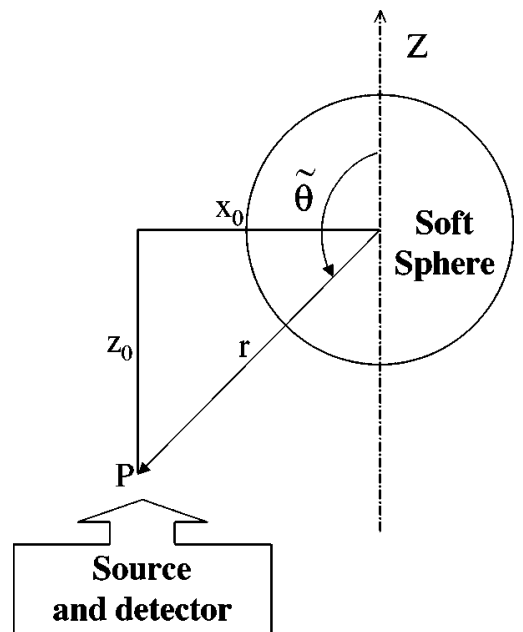

FIG. 2. Backscattered scheme due to pulse incident off center by a distance $x=-x_{0}$.

$$
\begin{aligned}
\Psi_{\mathrm{AXW}}^{s}(r, \theta, \phi, t) & \\
= & \frac{i 4 \pi^{2}}{c \rho_{0}} \int_{0}^{\infty} d k k^{q} e^{-k a} e^{i k c t} \sum_{n=0}^{\infty}-(-i)^{n}(2 n+1) \\
& \times \frac{j_{n}^{\prime}(k R)+i \alpha_{n} j_{n}(k R)}{h_{n}^{(2) \prime}(k R)+i \alpha_{n} h_{n}^{(2)}(k R)} h_{n}^{(2)}(k r) \sum_{m=0}^{n}(i)^{m} \epsilon_{m} \\
& \times \frac{(n-m) !}{(n-m) !} P_{n}^{m}(\cos \tilde{\theta}) P_{n}^{m}(\cos \xi) J_{m}\left(k x_{0} \sin \xi\right),
\end{aligned}
$$

with the backscattered angle defined as $\tilde{\theta}=(\pi / 2)$ $+\cos ^{-1}\left(x_{0} / r\right)$, as shown in Fig. 2. One should note that, in the configuration considered in Fig. 2, the detector point is placed close to the generator of the AXW. This off-axis detection position results in the two summations over $m$ and $n$ in Eq. (15). In addition, due to the lack of azimuthal symmetry the integration over $\phi_{k}$ yields the $J_{m}\left(k x_{0} \sin \xi\right)$ term.

\section{ANALYSIS OF THE BACKSCATTERED SPECTRUM}

The scattered pressure pulse, calculated from Eq. (2), is given by

$$
\begin{aligned}
p_{\mathrm{AXW}}^{s}(r, \theta, \phi, t) & \\
= & 4 \pi^{2} \int_{0}^{\infty} d k k^{q+1} e^{-k a} e^{i k c t} \sum_{n=0}^{\infty}-(-i)^{n}(2 n+1) \\
& \times \frac{j_{n}^{\prime}(k R)+i \alpha_{n} j_{n}(k R)}{h_{n}^{(2) \prime}(k R)+i \alpha_{n} h_{n}^{(2)}(k R)} h_{n}^{(2)}(k r) P_{n}(\cos \theta) \\
& \times P_{n}(\cos \xi)
\end{aligned}
$$

for the on-axis incidence case, and by 
TABLE I. Tabulated bulk modulus, density, and the calculated speed of sound propagation in different materials. (Ref. 10).

\begin{tabular}{|c|c|c|c|}
\hline Material & $\begin{array}{c}\text { Bulk modulus } \\
B \\
\left(10^{11} \mathrm{~N} / \mathrm{m}^{2}\right)\end{array}$ & $\begin{array}{c}\text { density } \\
\rho_{e} \\
\left(10^{3} \mathrm{~kg} / \mathrm{m}^{3}\right)\end{array}$ & $\begin{array}{c}\text { Speed } \\
c_{e}=\sqrt{B / \rho_{e}} \\
(\mathrm{~m} / \mathrm{s})\end{array}$ \\
\hline Titanium & 1.051 & 4.51 & 4827.40 \\
\hline Manganese & 0.596 & 7.47 & 2824.64 \\
\hline Nickel & 1.860 & 8.91 & 4568.96 \\
\hline Molybdenum & 2.725 & 10.22 & 5163.66 \\
\hline Aluminum & 0.722 & 2.70 & 5171.14 \\
\hline Chromium & 1.901 & 7.19 & 5141.93 \\
\hline Copper & 1.370 & 8.93 & 3916.83 \\
\hline Iron & 1.683 & 7.87 & 4624.39 \\
\hline Lead & 0.430 & 11.34 & 1947.28 \\
\hline Silver & 1.007 & 10.50 & 3096.85 \\
\hline
\end{tabular}

$$
\begin{aligned}
p_{\mathrm{AXW}}^{s}( & r, \theta, \phi, t) \\
= & 4 \pi^{2} \int_{0}^{\infty} d k k^{q+1} e^{-k a} e^{i k c t} \sum_{n=0}^{\infty}-(-i)^{n}(2 n+1) \\
& \times \frac{j_{n}^{\prime}(k R)+i \alpha_{n} j_{n}(k R)}{h_{n}^{(2) \prime}(k R)+i \alpha_{n} h_{n}^{(2)}(k R)} h_{n}^{(2)}(k r) \sum_{m=0}^{n}(i)^{m} \epsilon_{m} \\
& \times \frac{(n-m) !}{(n-m) !} P_{n}^{m}(\cos \tilde{\theta}) P_{n}^{m}(\cos \xi) J_{m}\left(k x_{0} \sin \xi\right)
\end{aligned}
$$

for the off-axis incidence case.

Our aim in this section is to examine some general features of the spectra of backscattered fields from various types of spheres. In particular, we will consider the absolute spectrum, which is the square root of the sum of the squares of the real and imaginary parts of the integrand in Eq. (16) or (17). We assume that the spheres are made of materials with the properties listed in Table I. A comparison of Figs. 3(a) and 3(b) shows that spheres made of $\mathrm{Mn}$ and $\mathrm{Pb}$ have different backscattered spectra when placed in water, whereas there is a small difference when they are situated in air. Examining the expression given in Eq. (13), we notice that $\alpha_{n}$ $\rightarrow 0$ if the ratio $\rho_{0} c / \rho_{e} c_{e}$ is very small. For spheres placed in air, $\rho_{0}=1.2 \mathrm{~kg} / \mathrm{m}^{3}$ and $c=350 \mathrm{~m} / \mathrm{s}$, while for spheres in water, $\rho_{0}=1.0 \times 10^{3} \mathrm{~kg} / \mathrm{m}^{3}$ and $c=1500 \mathrm{~m} / \mathrm{s}$ approximately. Thus, the product $\rho_{0} c$ is approximately equal to 400 for air and $1.5 \times 10^{6}$ for water. All the materials of Table I have $\rho_{e} c_{e} \approx(10-50) \times 10^{6}$; therefore $\alpha_{n} \rightarrow 0$ for spheres placed in air. In this case, the coefficient given in Eq. (13) reduces to that of a rigid sphere. For the same spheres immersed in water $\alpha_{n}$ cannot be neglected. Since $\alpha_{n}$ depends on $k_{e}, c_{e}$, and $\rho_{e}$, we expect that these quantities could be extracted from the backscattered AXW spectrum. This explains the reason for Fig. 3 exhibiting different backscattered AXW spectra when the $\mathrm{Mn}$ and $\mathrm{Pb}$ spheres are immersed in water.

In Figs. 4-6, we provide the backscattered spectra for spheres made of four different materials, namely, Ti, Mn, Ni, and Mo. These spectra are calculated for radii of 30, 35, and $40 \mathrm{~mm}$. From these plots, we specify three parameters: $\Delta k_{\mathrm{av}}$, $A$, and $\Delta k_{\mathrm{av}}^{\prime}$, which are the average spacing of the spectral dips at low frequency, the amplitude of the first peak, and the average spacing of the spectral dips over the entire spectrum,

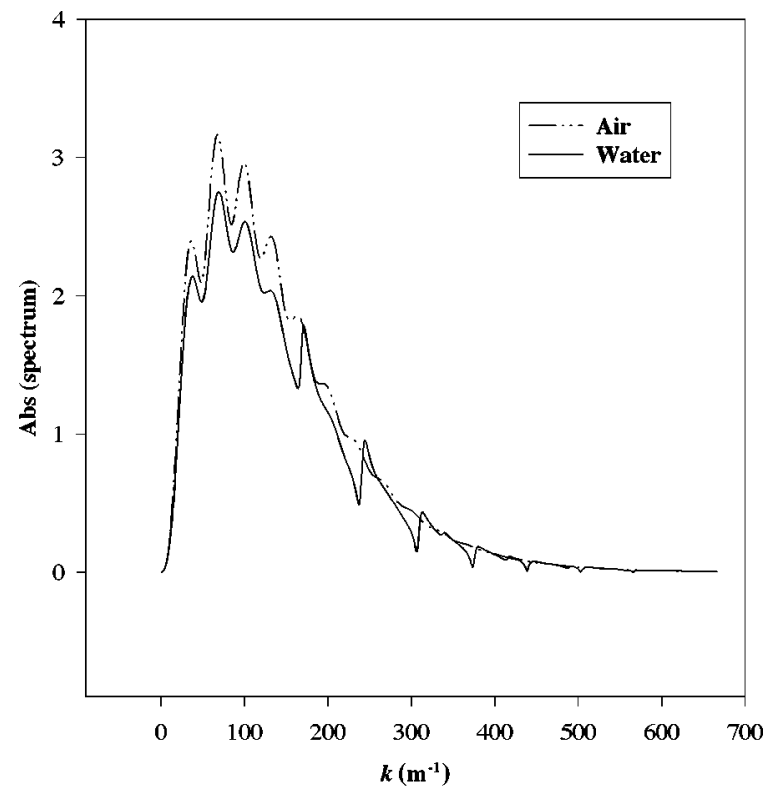

(a)

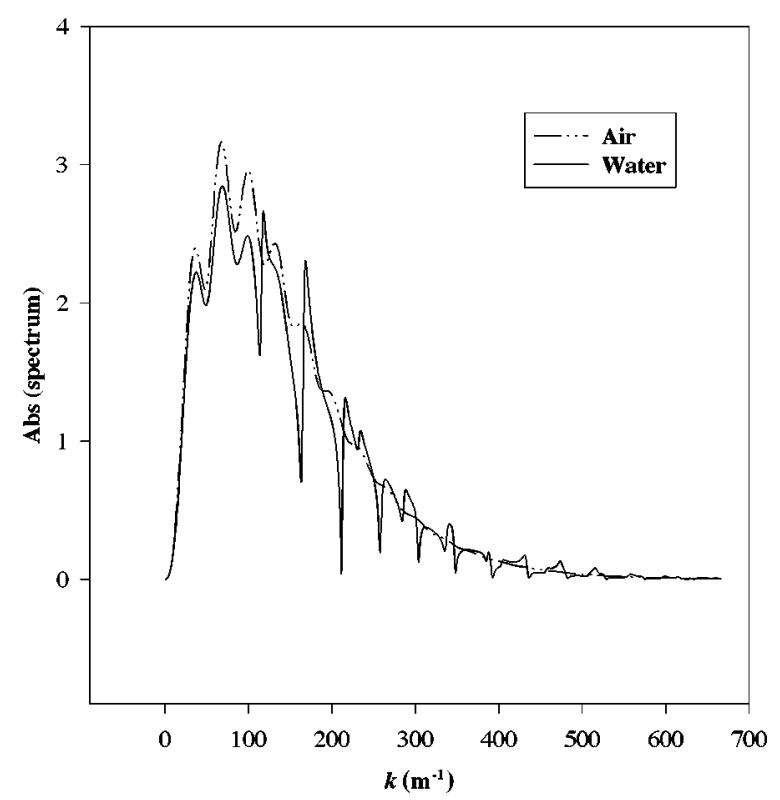

(b)

FIG. 3. Backscattered spectra by (a) Mn sphere and (b) $\mathrm{Pb}$ sphere having $R=35 \mathrm{~mm}$, placed in air and immersed in water, for $r=150 \mathrm{~mm}, a$ $=15 \mathrm{~mm}$, and $\xi=2^{\circ}$.

respectively. Figures $4-6$ show that $\Delta k_{\mathrm{av}}$ varies with the radius of the scattering sphere irrespective of the material. In addition, $A$ and $\Delta k_{\mathrm{av}}^{\prime}$ change with the density of the sphere and the speed of wave propagation inside the sphere, respectively. However, it is important to note that $A$ not only depends on the density of the material but also the radius, the observation distance, and the power of the received signal, which is proportional to the power of the source.

In Ref. 9, it has been demonstrated that more details appear in the backscattered spectrum if the radius of the sphere becomes larger, keeping the pulse width constant. In addition, as the radius of the sphere increases, the average spacing of the spectral dips becomes smaller and the ampli- 


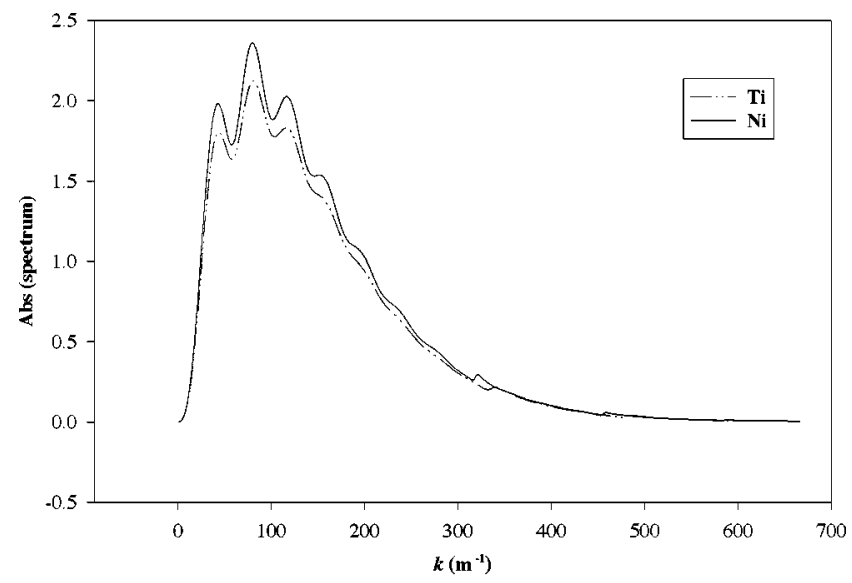

(a)

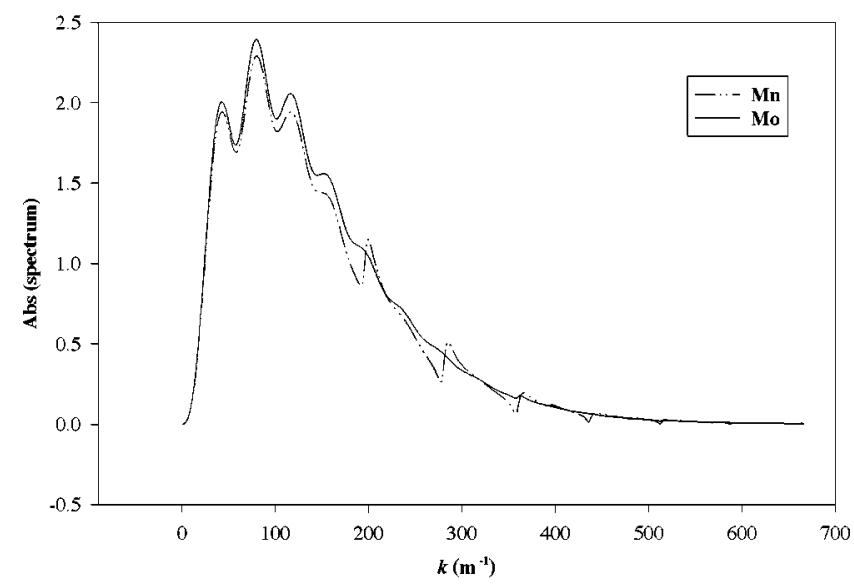

(b)

FIG. 4. Backscattered spectra of (a) Ti and Ni, (b) Mn and Mo spheres having radii $R=30 \mathrm{~mm}$, immersed in water for $r=150 \mathrm{~mm}, a=15 \mathrm{~mm}$, and $\xi=2^{\circ}$.

tude of the spectrum becomes larger. The low-frequency portion of the backscattered spectrum (approximately up to 30 $\mathrm{kHz}$ ) for a nonrigid sphere is similar to that of a rigid sphere. Nevertheless, in the high-frequency portion of the spectrum, one notices more pronounced dips for nonrigid spheres. ${ }^{3,9}$ Figure 3 shows that the average spacing of the spectral dips in the low-frequency range is almost identical for $\mathrm{Mn}$ and $\mathrm{Pb}$ spheres placed in water. However, the average spacing between the dips in the high-frequency ranges are different. Using the backscattered spectra of AXW pulses resulting from spheres of different materials placed in water, we can extract information about the size of the spheres if we have a "calibration" curve that relates the average spacing of the spectral dips calculated from the low-frequency range and the radii of the spheres. In Fig. 7, we provide such calibration curves using the low-frequency dip separations for the four materials whose spectra are provided in Figs. 4-6; namely, Ti, Mn, Ni, and Mo. The calibration curves provided in Fig. 7 for the four materials are very close to each other. Consequently, we can choose to start with an arbitrary calibration curve that does not depend on the material. Such an arbitrary "radius calibration" curve is shown in Fig. 8. This curve has been created using the average spacing of the spectral dips obtained from the low-frequency range of the back-

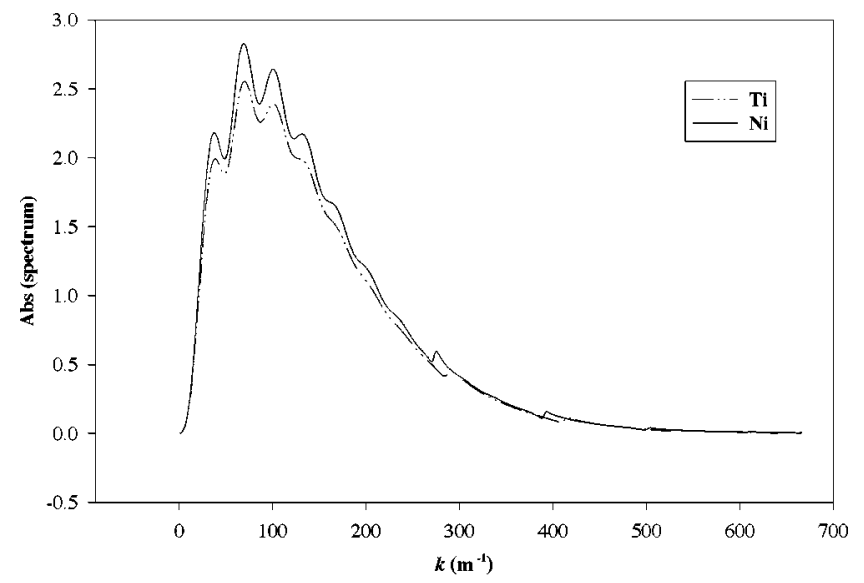

(a)

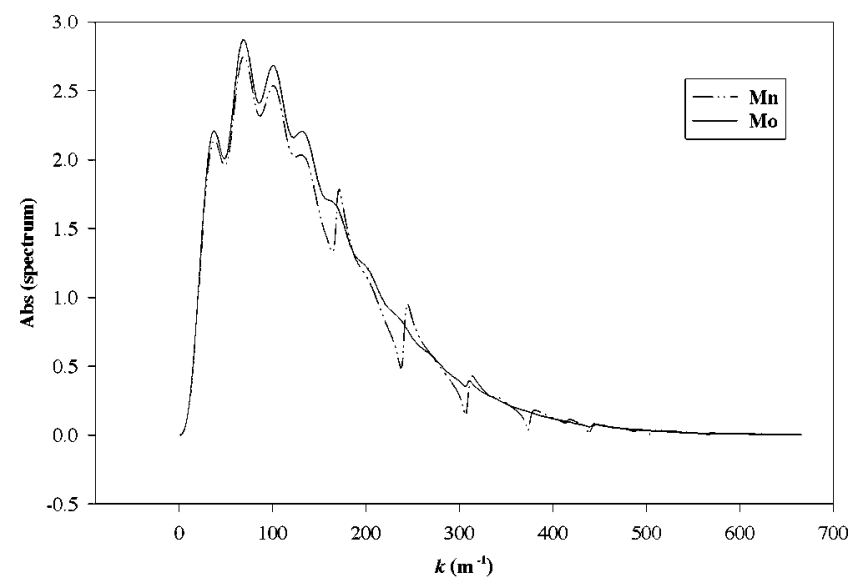

(b)

FIG. 5. Backscattered spectra of (a) Ti and Ni, (b) Mn and Mo spheres having radii $R=35 \mathrm{~mm}$, immersed in water for $r=150 \mathrm{~mm}, a=15 \mathrm{~mm}$, and $\xi=2^{\circ}$.

scattered spectra of three different spheres having radii equal to 30,40 , and $50 \mathrm{~mm}$. The spectra of the scattered fields for these radii are evaluated for $r=150 \mathrm{~mm}, a=15 \mathrm{~mm}, \rho_{0}$ $=1.0 \times 10^{3} \mathrm{~kg} / \mathrm{m}^{3}, \quad \rho_{e}=7.8 \times 10^{3} \mathrm{~kg} / \mathrm{m}^{3}, \quad c=1500 \mathrm{~m} / \mathrm{s}$, and $c_{e}=3000 \mathrm{~m} / \mathrm{s}$. The points are plotted using MS Excel and a trend line is drawn, yielding the equation $R$ $=1041.1\left(\Delta k_{\mathrm{av}}\right)^{-0.937}$ for the calibration curve. In all calculations, we have used the spectra derived from Eq. (16), with the parameter values $a=15 \mathrm{~mm}, q=0$, and $\xi=2^{\circ}$.

For scattering spheres having the same radii and situated at the same observation distance, the simulated backscattered spectra of spheres made of different materials show that each material gives a different amplitude. However, the amplitudes are not only dependent on the density of the material but also the radius of the sphere, the observation distance, and the power of the received signal. The latter is obviously proportional to the power of the source. Therefore, if we know the power of the source and the observation distance, we can relate the density of the material to the amplitude of the backscattered spectrum for spheres having different radii. In particular, we have observed that the relation between the amplitude and the density takes the form of $A \propto 1 / \sqrt{\rho_{e}}$, where $A$ is the amplitude and $\rho_{e}$ is the density of the material of the sphere. In addition, we argue that the amplitude of the first 


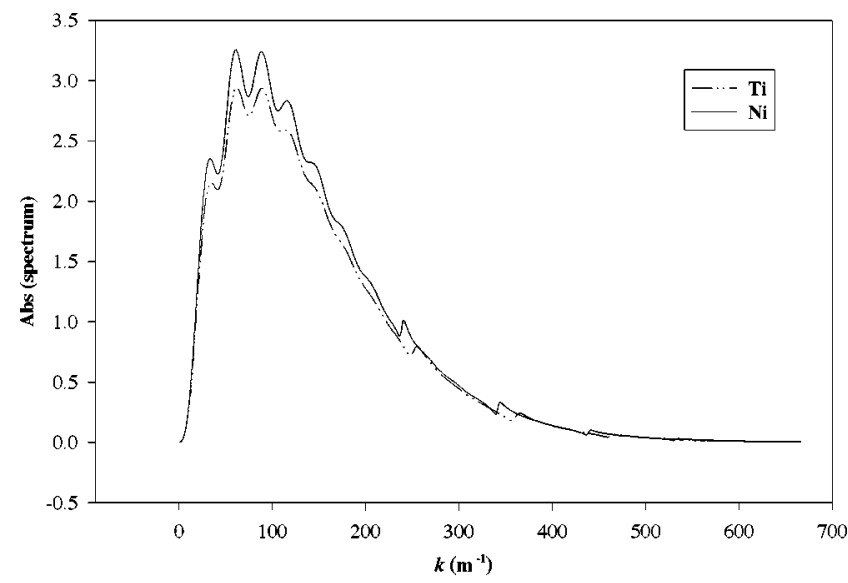

(a)

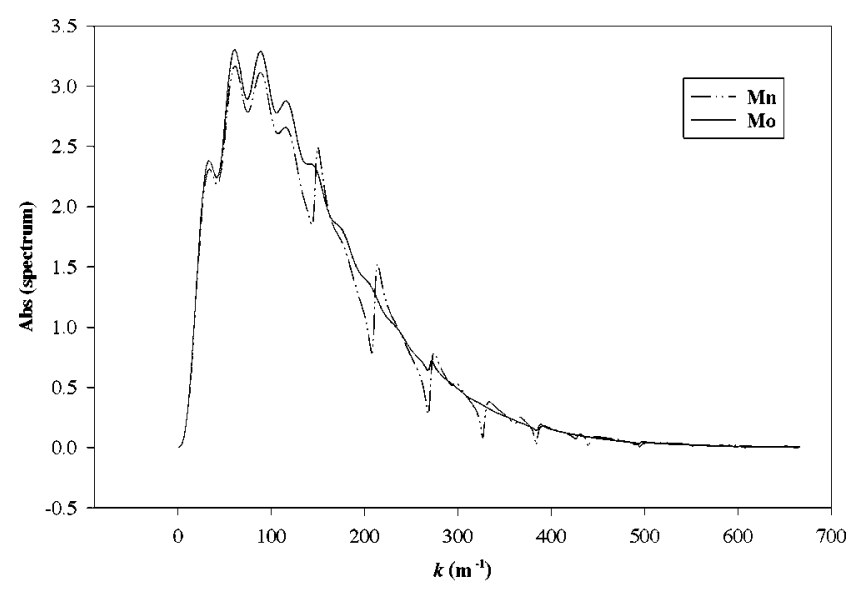

(b)

FIG. 6. Backscattered spectra of (a) $\mathrm{Ti}$ and $\mathrm{Ni}$, (b) $\mathrm{Mn}$ and Mo spheres having radii $R=40 \mathrm{~mm}$, immersed in water for $r=150 \mathrm{~mm}, a=15 \mathrm{~mm}$, and $\xi=2^{\circ}$.

peak in the spectrum yields acceptable results. Figure 9 provides calibration curves relating the amplitudes of the first peaks in the backscattered spectra of four different materials ( $\mathrm{Ti}, \mathrm{Mn}, \mathrm{Ni}$, and $\mathrm{Mo}$ ) to their densities for radii of 30,35 , and $40 \mathrm{~mm}$. The data are plotted using MS Excel and trend

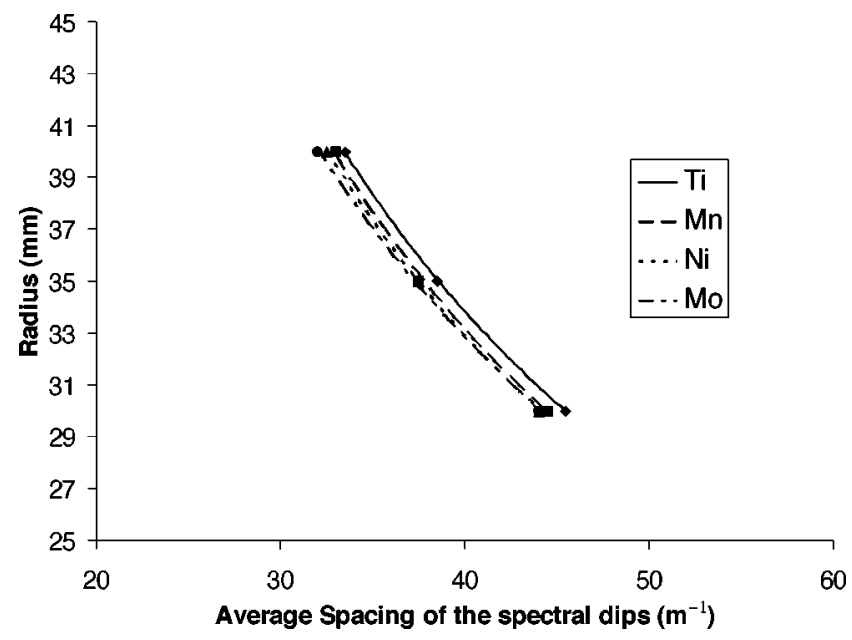

FIG. 7. Calibration curves relating the radius of the sphere to the average spacing of the spectral dips by four different materials, Ti, Mn, Ni, and Mo, immersed in water for $r=150 \mathrm{~mm}, a=15 \mathrm{~mm}$, and $\xi=2^{\circ}$.

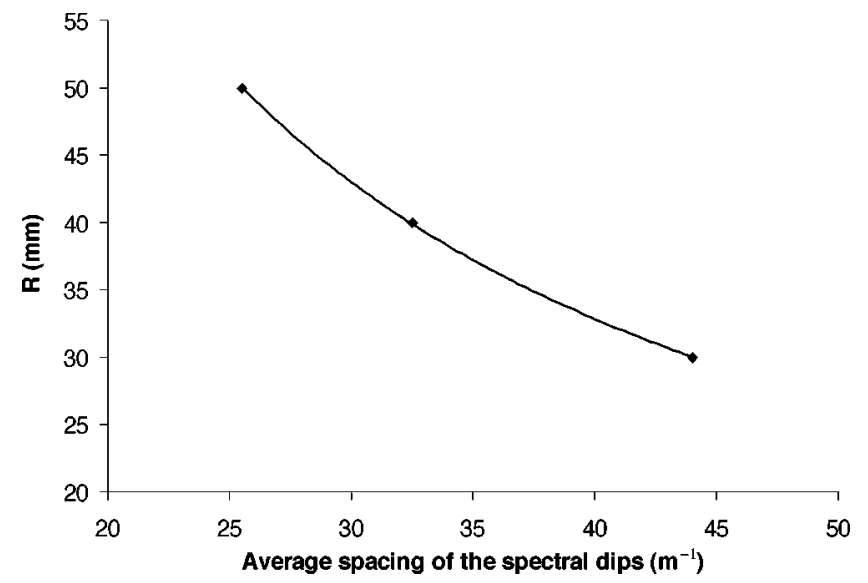

FIG. 8. Calibration curve between the radius of the sphere and average spacing of the spectral dips of the backscattered spectrum for $r=150 \mathrm{~mm}$, $\rho_{0}=1.0 \times 10^{3} \mathrm{~kg} / \mathrm{m}^{3}, c=1500 \mathrm{~m} / \mathrm{s}, \quad \rho_{e}=7.8 \times 10^{3} \mathrm{~kg} / \mathrm{m}^{3}, \quad c_{e}=3000 \mathrm{~m} / \mathrm{s}, a$ $=15 \mathrm{~mm}$, and $\xi=2^{\circ}$.

lines are drawn to give mathematical forms for these curves. For example, the equation of the line for $R=35 \mathrm{~mm}, r$ $=150 \mathrm{~mm}, \quad a=15 \mathrm{~mm}, \quad \rho=1.0 \times 10^{3} \mathrm{~kg} / \mathrm{m}^{3}, \quad$ and $\quad c_{e}$ $=1500 \mathrm{~m} / \mathrm{s}$ is given by $1 / \sqrt{\rho_{e}}=-0.0228 A+0.0603$. In Fig. 10 , we present typical calibration curves between density and amplitude at different observation distances. The relationship between the amplitude and observation distance, shown in Fig. 11, is the expected $A \propto 1 / r$ as indicated by the trend lines of the MS Excel plot.

Although varying the speed of wave propagation inside the sphere affects the average spacing of the spectral dips in the high-frequency range, we can deduce from Fig. 7 that the speed also has a small effect on the low-frequency range because the four curves do not overlap completely. Therefore, we recommend that the average spacing of the spectral dips of the whole backscattered spectrum be calculated when doing the calibration curve for the speed of sound in the material. Figure 12 provides a typical calibration curve for the average spacing of the spectral dips of the entire spectrum with the speed of wave propagation inside the sphere

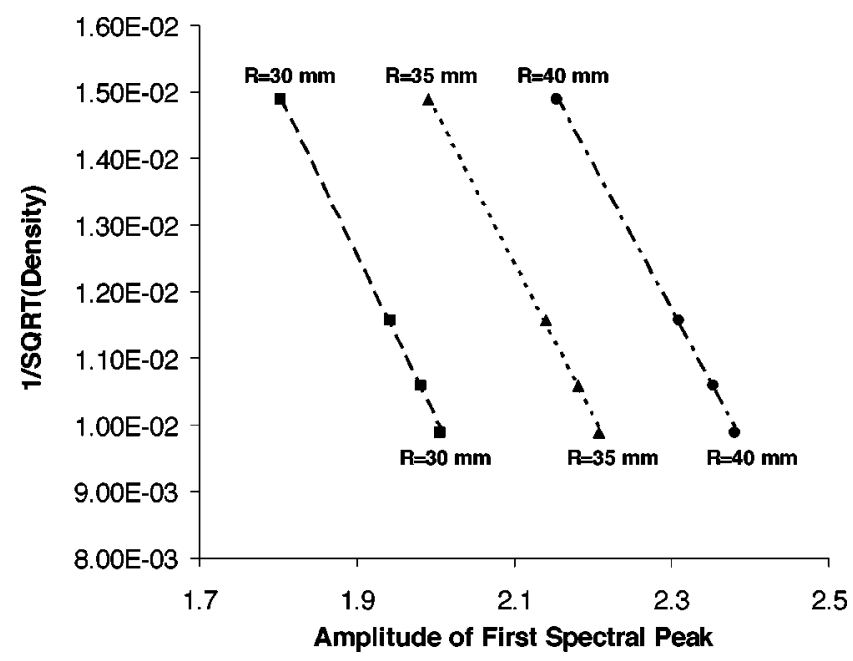

FIG. 9. Calibration curves relating the density of the material to the amplitude of the backscattered spectrum for different radii, $r=150 \mathrm{~mm}, \rho_{0}=1.0$ $\times 10^{3} \mathrm{~kg} / \mathrm{m}^{3}, c=1500 \mathrm{~m} / \mathrm{s}, a=15 \mathrm{~mm}$, and $\xi=2^{\circ}$. 


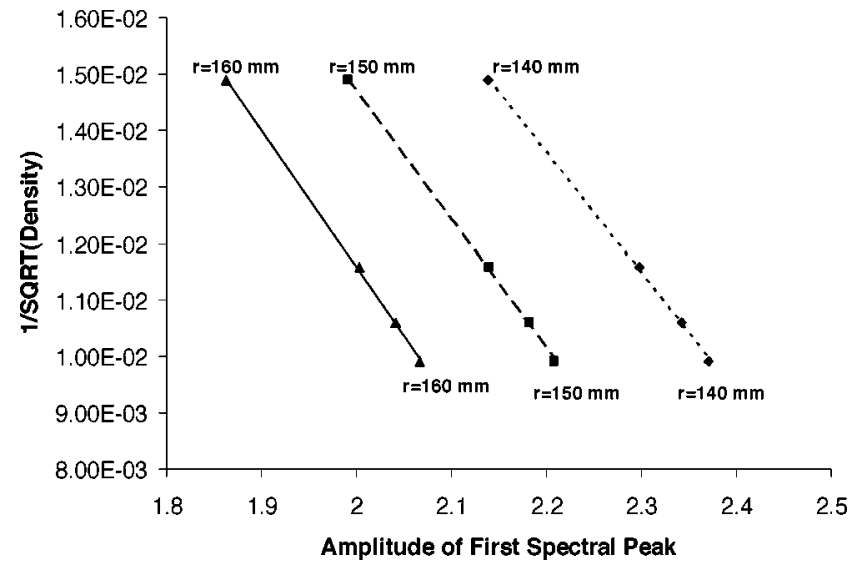

FIG. 10. Calibration curves relating the density of the material to the amplitude of the backscattered spectrum for different observation distances, $R=35 \mathrm{~mm}, \rho_{0}=1.0 \times 10^{3} \mathrm{~kg} / \mathrm{m}^{3}, c=1500 \mathrm{~m} / \mathrm{s}, a=15 \mathrm{~mm}$, and $\xi=2^{\circ}$.

for different radii. Again, we have used the backscattered spectra of $\mathrm{Ti}, \mathrm{Mn}, \mathrm{Ni}$, and Mo with the same aforementioned pulse parameters.

\section{PROPOSED IDENTIFICATION SCHEME}

In the preceding section, we established calibration relations for the radius, density, and speed of wave propagation, using the backscattered spectra produced by spheres made of four different materials: $\mathrm{Ti}, \mathrm{Mn}, \mathrm{Ni}$, and Mo. In this section, we are going to show how we can use these relations to identify the sizes and material properties of unknown spherical scatterers using their simulated backscattered spectra. Making use of the results of Sec. IV, we can outline a procedure for identifying the size and material of an unknown spherical scatterer. First, we can estimate the radius using an arbitrary "radius calibration" curve, which is almost material independent (cf. Figs. 7 and 8). This arbitrary calibration curve relates the average spacing of the spectral dips at low frequencies and the radius of the sphere. Using the estimated value of the radius, we choose the appropriate calibration curve between the average spectral dip spacing obtained from the entire spectrum and the speed to determine the speed of wave propagation inside the sphere. Similarly, using

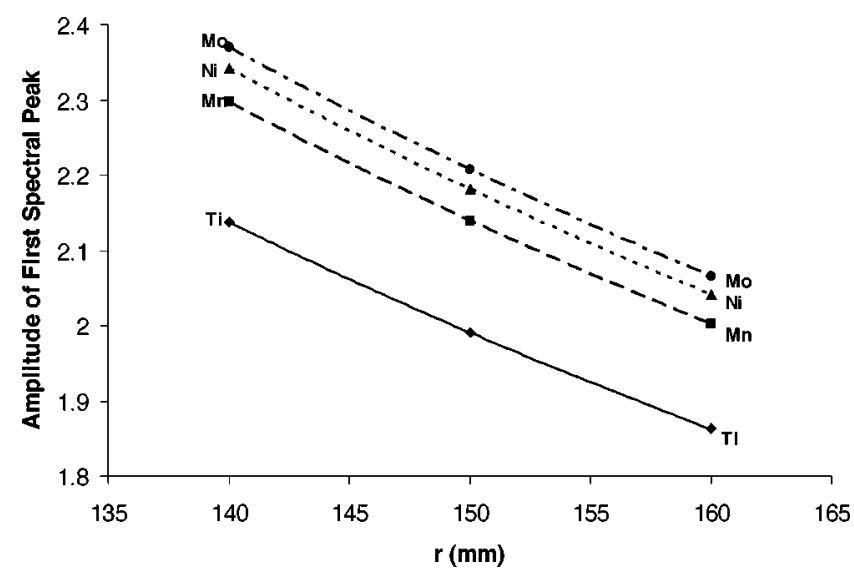

FIG. 11. Calibration curves relating the amplitude of the backscattered spectrum to the observation distance for different materials, $\mathrm{Ti}, \mathrm{Mn}, \mathrm{Ni}$, and $\mathrm{Mo}$, immersed in water for $R=35 \mathrm{~mm}, a=15 \mathrm{~mm}$, and $\xi=2^{\circ}$.

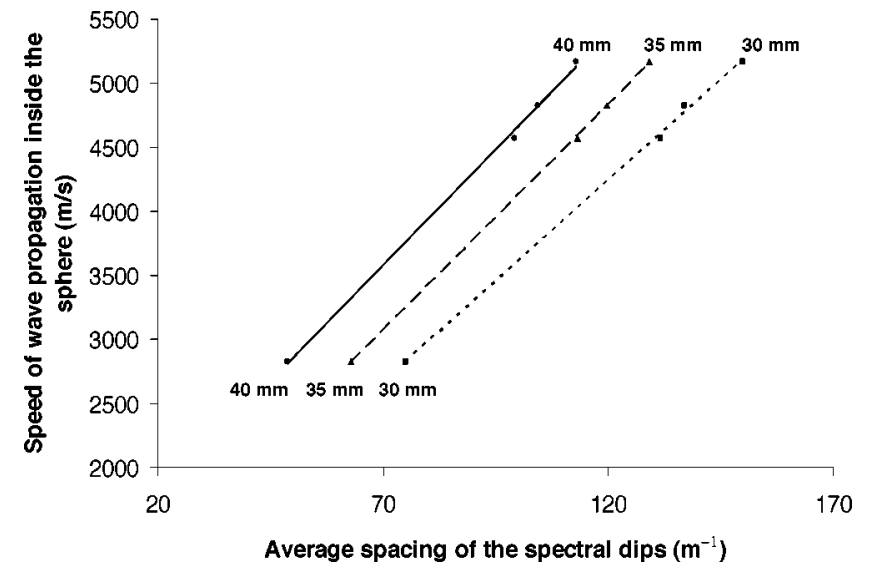

FIG. 12. Calibration curves relating the speed of wave propagation inside of the sphere to the average spacing of the spectral dips of the backscattered spectrum for different radii, $\quad r=150 \mathrm{~mm}, \quad \rho_{0}=1.0 \times 10^{3} \mathrm{~kg} / \mathrm{m}^{3}, \quad c$ $=1500 \mathrm{~m} / \mathrm{s}, a=15 \mathrm{~mm}$, and $\xi=2^{\circ}$.

the estimated radius to choose the correct calibration curve relating the amplitude and density of the material, we identify the density of the scatterer.

Consider the backscattered spectra for $35-\mathrm{mm}$ spheres made of the following materials: $\mathrm{Al}, \mathrm{Cr}, \mathrm{Cu}, \mathrm{Fe}, \mathrm{Pb}$, and $\mathrm{Ag}$

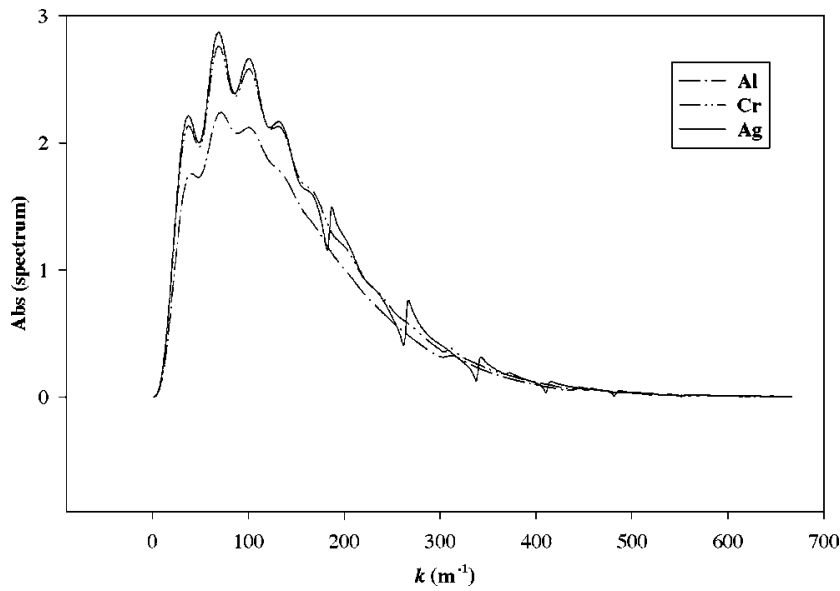

(a)

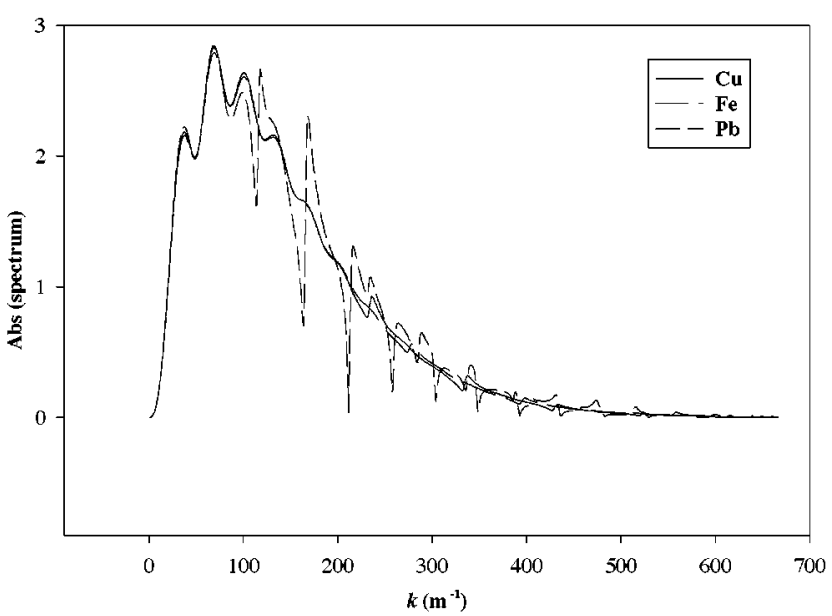

(b)

FIG. 13. Backscattered spectra for (a) $\mathrm{Al}, \mathrm{Cr}, \mathrm{Ag}$, (b) $\mathrm{Cu}, \mathrm{Fe}, \mathrm{Pb}$ for $r$ $=150 \mathrm{~mm}, \rho_{0}=1.0 \times 10^{3} \mathrm{~kg} / \mathrm{m}^{3}, c=1500 \mathrm{~m} / \mathrm{s}, a=15 \mathrm{~mm}$, and $\xi=2^{\circ}$. 
TABLE II. The estimated and actual radii of the spheres as have been calculated from the average spacing of the spectral dips of the simulated backscattered spectra obtained for different materials due to on-center incidence, as well as for off-center incidence at distances $x_{0}=15 \mathrm{~mm}$ and $x_{0}=45 \mathrm{~mm}$.

\begin{tabular}{|c|c|c|c|c|c|c|c|c|c|}
\hline \multirow[b]{3}{*}{ Material } & & & & \multicolumn{6}{|c|}{ Off center } \\
\hline & \multicolumn{3}{|c|}{ On center } & \multicolumn{3}{|c|}{$x_{0}=15 \mathrm{~mm}$} & \multicolumn{3}{|c|}{$x_{0}=45 \mathrm{~mm}$} \\
\hline & $\begin{array}{c}\Delta k_{\mathrm{av}} \\
\left(\mathrm{m}^{-1}\right)\end{array}$ & $\begin{array}{c}\text { Est. } \\
R \\
(\mathrm{~mm})\end{array}$ & $\begin{array}{c}\% \\
\text { error }\end{array}$ & $\begin{array}{c}\Delta k_{\mathrm{av}} \\
\left(\mathrm{m}^{-1}\right)\end{array}$ & $\begin{array}{c}\text { Est. } \\
R \\
(\mathrm{~mm})\end{array}$ & $\begin{array}{c}\% \\
\text { error }\end{array}$ & $\begin{array}{c}\Delta k_{\mathrm{av}} \\
\left(\mathrm{m}^{-1}\right)\end{array}$ & $\begin{array}{c}\text { Est. } \\
R \\
(\mathrm{~mm})\end{array}$ & $\begin{array}{c}\% \\
\text { error }\end{array}$ \\
\hline $\mathrm{Al}$ & 40.50 & 32.45 & 7.29 & 41.00 & 32.06 & 8.41 & 43.00 & 30.56 & 12.68 \\
\hline $\mathrm{Cr}$ & 37.50 & 35.06 & 0.17 & 38.00 & 34.59 & 1.16 & 39.00 & 33.7 & 3.70 \\
\hline $\mathrm{Cu}$ & 37.25 & 35.29 & 0.83 & 37.50 & 35.06 & 0.17 & 38.50 & 34.14 & 2.45 \\
\hline $\mathrm{Fe}$ & 37.50 & 35.06 & 0.17 & 37.75 & 34.82 & 0.50 & 39.00 & 33.70 & 3.70 \\
\hline $\mathrm{Pb}$ & 37.50 & 35.06 & 0.17 & 38.00 & 34.59 & 1.16 & 39.50 & 33.28 & 4.92 \\
\hline $\mathrm{Ag}$ & 37.00 & 35.53 & 1.51 & 37.00 & 35.53 & 1.51 & 38.50 & 34.14 & 2.45 \\
\hline
\end{tabular}

due to an AXW pulse incident on their centers. From Fig. 13, we calculate the average spacing of spectral dips at low frequencies (up to approximately $35 \mathrm{kHz}$ ). Using these values in the arbitrary "radius calibration" curve given in Fig. 8, we can estimate their radii, obtaining values that are approximately equal to $35 \mathrm{~mm}$ (cf. Table II). Subsequently, we obtain from the backscattered spectra the amplitudes of the first peaks and the average spacings of the spectral dips of the entire spectrum. According to the estimated radii, we use the appropriate density and speed calibration curves (cf. Figs. 9 and 12) to determine the density of the material and speed of sound propagation inside the spheres. Table II shows the estimated radii of the different materials and the percentage error. The radii are estimated for on-center and off-center incidences. For on-center incidence, the estimated radii for all the materials have accuracy levels better than $98 \%$, except for $\mathrm{Al}$, which has an accuracy level of $92 \%$ because its backscattered spectrum does not contain enough detail. In order to obtain better results for $\mathrm{Al}$, we should use a smaller pulse width. This is equivalent to having a wider spectral bandwidth. However, the pulse width should not be smaller than five times the radius. For off-center incidence, the errors in the estimated radii do not vary much when $x_{0}=15 \mathrm{~mm}$. However, as the axis of propagation of the $\mathrm{X}$ wave moves out of the body of the sphere (at $x_{0}=45 \mathrm{~mm}$ ), the errors in the estimated radii start increasing, especially for $\mathrm{Pb}$ for which the percentage error increases to $5 \%$ and for $\mathrm{Al}$ it rises to $12.63 \%$.

Table III contains estimates of the densities of the scat- tering spheres. It can be seen that for the on-center case, the percentage errors in estimating the densities are smaller than $6 \%$ for all materials except for $\mathrm{Al}$ and $\mathrm{Ag}$ that have approximately $12.2 \%$ and $8.2 \%$ errors, respectively. Due to the relationship (amplitude) $\propto 1 / \sqrt{(\text { density })}$, the determination of the density is very sensitive to errors in the estimated radius of the sphere. Therefore, errors for 45-mm off-center incidence climb up to unacceptable values, e.g., we obtain $39 \%$ error in the estimated density of Al. Other spheres have density estimates exhibiting errors that are greater than $9 \%$. The $15-\mathrm{mm}$ off-center incidence yields acceptable estimates with errors that are smaller than $10 \%$, except for $\mathrm{Al}$. This indicates that the density values determined using this method are reliable only when the focused part of the incident pulse hits the scattering sphere. The sound speed in the material of the sphere is determined from the average dip spacings over the whole spectrum. In Table IV, we provide estimates of the sound speeds for on- and off-center incidences. For the three cases under consideration, error margins smaller than 5\% are achieved except for $\mathrm{Al}$ that has an on-center percentage error of $6.7 \%$ and climbs up to $11.1 \%$ for the 45 -mm off-incidence case. Thus, it is seen that the percentage errors in estimating the speed of wave propagation are small whether the pulse is incident on center or off center. Our discussion shows that the information extracted from the separation of dips $\left(\Delta k_{\mathrm{av}}\right.$ or $\Delta k_{\mathrm{av}}^{\prime}$ ) is highly reliable and yields good estimates of the radii and wave speeds for the spheres. On the other hand, the accuracy by which the density is determined is highly sensi-

TABLE III. The estimated densities of the spheres for $R=35 \mathrm{~mm}$ as calculated from the amplitudes of the simulated backscattered spectra obtained for different materials due to on-center incidence as well as off-center incidence by distances $x_{0}=15 \mathrm{~mm}$ and $x_{0}=45 \mathrm{~mm}$.

\begin{tabular}{|c|c|c|c|c|c|c|c|c|c|}
\hline \multirow[b]{3}{*}{ Material } & \multirow{2}{*}{\multicolumn{3}{|c|}{ On center }} & \multicolumn{6}{|c|}{ Off center } \\
\hline & & & & \multicolumn{3}{|c|}{$x_{0}=15 \mathrm{~mm}$} & \multicolumn{3}{|c|}{$x_{0}=45 \mathrm{~mm}$} \\
\hline & A & $\begin{array}{c}\text { Est. } \\
\rho_{e} \\
\left(\mathrm{~kg} / \mathrm{m}^{3}\right)\end{array}$ & $\begin{array}{c}\% \\
\text { error }\end{array}$ & $A$ & $\begin{array}{c}\text { Est. } \\
\rho_{e} \\
\left(\mathrm{~kg} / \mathrm{m}^{3}\right.\end{array}$ & $\begin{array}{c}\% \\
\text { error }\end{array}$ & A & $\begin{array}{c}\text { Est. } \\
\rho_{e} \\
\left(\mathrm{~kg} / \mathrm{m}^{3}\right)\end{array}$ & $\begin{array}{l}\% \\
\text { error }\end{array}$ \\
\hline $\mathrm{Al}$ & 1.7587 & $3.03 \times 10^{3}$ & 12.2 & 1.7601 & $3.13 \times 10^{3}$ & 16.1 & 1.7719 & $3.76 \times 10^{3}$ & 39.3 \\
\hline $\mathrm{Cr}$ & 2.1334 & $7.36 \times 10^{3}$ & 2.3 & 2.1338 & $7.78 \times 10^{3}$ & 9.5 & 2.1373 & $9.4 \times 10^{3}$ & 9.7 \\
\hline $\mathrm{Cu}$ & 2.1817 & $8.45 \times 10^{3}$ & 5.3 & 2.1819 & $8.98 \times 10^{3}$ & 0.6 & 2.1841 & $1.07 \times 10^{4}$ & 19.8 \\
\hline $\mathrm{Fe}$ & 2.1546 & $8.01 \times 10^{3}$ & 1.7 & 2.1549 & $8.16 \times 10^{3}$ & 3.72 & 2.1579 & $1.03 \times 10^{4}$ & 30.9 \\
\hline $\mathrm{Pb}$ & 2.2216 & $1.07 \times 10^{4}$ & 4.9 & 2.2217 & $1.15 \times 10^{4}$ & 1.4 & 2.2225 & $1.47 \times 10^{4}$ & 29.6 \\
\hline $\mathrm{Ag}$ & 2.2120 & $9.64 \times 10^{3}$ & 8.2 & 2.2121 & $9.64 \times 10^{3}$ & 8.2 & 2.2137 & $1.24 \times 10^{4}$ & 18.1 \\
\hline
\end{tabular}


TABLE IV. The estimated acoustic speeds of propagation inside the spheres for $R=35 \mathrm{~mm}$ as have been calculated from the average spacing of the spectral dips of the backscattered spectra obtained for different materials due to incidence off center by a distance to on-center incidence as well as off-center incidence by distances $x_{0}=15 \mathrm{~mm}$ and $x_{0}=45 \mathrm{~mm}$.

\begin{tabular}{|c|c|c|c|c|c|c|c|c|c|}
\hline \multirow[b]{3}{*}{ Material } & \multirow{2}{*}{\multicolumn{3}{|c|}{ On center }} & \multicolumn{6}{|c|}{ Off center } \\
\hline & & & & \multicolumn{3}{|c|}{$x_{0}=15 \mathrm{~mm}$} & \multicolumn{3}{|c|}{$x_{0}=45 \mathrm{~mm}$} \\
\hline & $\begin{array}{c}\Delta k_{\mathrm{av}}^{\prime} \\
\left(\mathrm{m}^{-1}\right)\end{array}$ & $\begin{array}{c}\text { Est. } \\
c_{e} \\
(\mathrm{~m} / \mathrm{s})\end{array}$ & $\begin{array}{l}\% \\
\text { error }\end{array}$ & $\begin{array}{c}\Delta k_{\mathrm{av}}^{\prime} \\
\left(\mathrm{m}^{-1}\right)\end{array}$ & $\begin{array}{c}\text { Est. } \\
c_{e} \\
(\mathrm{~m} / \mathrm{s})\end{array}$ & $\begin{array}{c}\% \\
\text { error }\end{array}$ & $\begin{array}{c}\Delta k_{\mathrm{av}}^{\prime} \\
\left(\mathrm{m}^{-1}\right)\end{array}$ & $\begin{array}{c}\text { Est. } \\
c_{e} \\
(\mathrm{~m} / \mathrm{s})\end{array}$ & $\begin{array}{c}\% \\
\text { error }\end{array}$ \\
\hline $\mathrm{Al}$ & 128.67 & 4824.48 & 6.7 & 128.83 & 4758.39 & 8.0 & 129.33 & 4595.40 & 11.1 \\
\hline $\mathrm{Cr}$ & 129.33 & 5162.10 & 0.39 & 129.33 & 5118.45 & 0.46 & 129.50 & 5005.17 & 2.66 \\
\hline $\mathrm{Cu}$ & 94.62 & 3943.13 & 0.67 & 94.62 & 3939.62 & 0.58 & 94.62 & 3831.04 & 2.19 \\
\hline $\mathrm{Fe}$ & 114.50 & 4639.71 & 0.33 & 114.67 & 4616.91 & 0.16 & 114.67 & 4488.26 & 2.94 \\
\hline $\mathrm{Pb}$ & 40.62 & 2037.29 & 4.62 & 40.62 & 1945.39 & 0.1 & 40.62 & 1883.56 & 3.27 \\
\hline $\mathrm{Ag}$ & 72.25 & 3139.45 & 1.38 & 72.12 & 3134.66 & 1.22 & 72.12 & 3037.62 & 1.91 \\
\hline
\end{tabular}

tive to any initial inaccurate estimation of the radius of the sphere.

\section{CONCLUDING REMARKS}

In this paper, we discussed the scattering of an AXW pulse by a sphere. We represented the incident AXW pulse as a spectral superposition over plane waves. Subsequently, we used the series solution of a plane wave scattered by a sphere in order to synthesize the scattered AXW pulse. The spectrum of the scattered field was shown to carry specific information regarding the size and material properties of the scattering sphere. We have undertaken a detailed study of the backscattered spectra for spheres having different radii and made out of diverse materials. Our work indicates that one can use the spectra of a few spheres of known materials and specified radii to generate a set of calibration curves. These curves can then be used to characterize unknown spherical scatterers. In particular, we can estimate the radius of the scatterer using an arbitrary calibration curve that does not depend on the material. Since the estimation of both the density and wave speed of a sphere are radius dependent, we utilize the estimated radius together with appropriate calibration curves for the density and the speed in order to identify the material. The effects of the observation distance and the power of the received signal, which is proportional to the power of the source used, must be taken into account in the process. To examine the robustness of our method, we have considered both on-center and off-center incidence.

The analysis used in this work indicates that it is advantageous to use ultra-wideband pulses for identification purposes because we can extract different pieces of information from various parts of their large spectral bandwidth. Specifically, the separation between the dips of the lower-frequency part of the spectrum is used to calculate the radius. The average spacings between the dips over the entire spectrum yield the wave speed in the material of the scatterer. The amplitude of the first peak determines the density of the material. Estimates of the radii, densities, and wave speeds of six unknown materials have been deduced using calibration curves generated for spheres made of four known materials. It has been shown that the percentage errors in our estimates are very low, except for a few exceptional cases. We have also demonstrated that the suggested procedure is robust even in the off-center incidence case, provided that the offcenter distance does not exceed the radius of the sphere if the density is estimated. On the other hand, the off-center distance can be increased to approximately $40 \%$ of the observation distance if the wave speed is evaluated. Although this work has been carried out for an incident $\mathrm{X}$ wave, we should point out that the advocated procedure for size identification and material characterization is not restricted to $\mathrm{X}$-wave pulses and can be applied to other types of ultra-wideband pulses.

Finally, we would like to point out that several theoretical studies of the scattering of electromagnetic $\mathrm{X}$ waves from conducting wedges and disks have been published. ${ }^{11,12}$ In these studies, the scattered fields were evaluated by combining the pulsed plane-wave representation of $\mathrm{X}$ waves ${ }^{1,2}$ with high-frequency asymptotic techniques. ${ }^{11,12}$ Contrary to the work presented in this paper, there was no attempt to use the backscattered fields in identifying any of the attributes of the scattering objects. It is of interest to extend the identification technique introduced in this paper such that the features of scatterers having different geometries could be determined from their backscattered spectrum. Another interesting situation is to be able to acoustically identify the attributes of a scattering object when it is immersed in water or is buried underground while the source of the AXW is situated in a second medium.

${ }^{1}$ A. M. Attiya, "Transverse (TE) electromagnetic X-waves: Propagation, scattering, diffraction and generation problems," Ph.D. thesis, Cairo University, 2001

${ }^{2}$ A. M. Attiya, E. A. El-Diwany, A. M. Shaarawi, and I. M. Besieris, "Reflection and transmission of X-waves in the presence of planarly layered media: The pulsed plane wave representation," Prog. Electromagn. Res. 30, 191-211 (2000).

${ }^{3}$ D. Power, R. Donnelly, and R. MacIsaac, "Spherical scattering of superpositions of localized waves," Phys. Rev. E 48, 1410-1417 (1993).

${ }^{4}$ R. W. Ziolkowski, "Localized transmission of electromagnetic energy," Phys. Rev. A 39, 2005-2033 (1989).

${ }^{5}$ J. Fagerholm, A. T. Friberg, J. Huttunen, D. P. Morgan, and M. M. Salomaa, "Angular-spectrum representation of nondiffracting X waves," Phys. Rev. E 54, 4347-4352 (1996).

${ }^{6}$ P. M. Morse, Vibration and Sound (McGraw-Hill, New York, 1948).

${ }^{7}$ A. M. Shaarawi, I. M. Besieris, A. M. Attiya, and E. A. El-Diwany, "Acoustic X-wave reflection and transmission at a planar interface: Spectral analysis," J. Acoust. Soc. Am. 107, 70-86 (2000).

${ }^{8}$ G. B. Arfkin, Mathematical Methods for Physicists (Academic, San Diego, 1995) 
${ }^{9}$ M. F. Z. Moawad, "Scattering of ultra-wideband X-wave pulses by a sphere," M.Sc. thesis, American University in Cairo, Egypt, 2002.

${ }^{10}$ C. Kittel, Introduction to Solid State Physics, 7th ed. (Wiley, New York, 1996).

${ }^{11}$ A. M. Attiya, E. A. El-Diwany, A. M. Shaarawi, and I. M. Besieris, "Dif- fraction of a transverse electric (TE) X wave by conducting objects," Prog. Electromagn. Res. 38, 167-198 (2003).

${ }^{12}$ A. M. Attiya, E. A. El-Diwany, A. M. Shaarawi, and I. M. Besieris, "Scattering of X-waves from a circular disk using a time domain incremental theory of diffraction," Prog. Electromagn. Res. 44, 103-129 (2003). 